\title{
E-FLOW PROJECT: Integração da Engenharia de Requisitos de Software com Business Process Management
}

\author{
Eduardo M. F. Jorge ${ }^{1}$, Marcus Vinícius A. Silva², Saulo Almeida ${ }^{3}$, Taluna Mendes ${ }^{4}$ \\ ${ }^{1}$ Centro Universitário da Bahia \\ ${ }^{2}$ Instituto Recôncavo de Tecnologia \\ ${ }^{3}$ Universidade Católica Do Salvador \\ ${ }^{4}$ Universidade Católica Do Salvador \\ emjorge@reconcavotecnologia.org.br, mvsilva@reconcavotecnologia.org.br \\ sauloandrade@gmail.com, taluna@nexen.com.br
}

\begin{abstract}
Resumo. A automatização dos processos é uma solução utilizada por muitas organizações com o objetivo de reduzir custos e contribuir na garantia qualidade em seus produtos e serviços. Entretanto, as que atuam com desenvolvimento de software muitas vezes deparam-se com a dificuldade de gerar, ao final do processo de desenvolvimento, um produto que atenda plenamente às necessidades de seus usuários e clientes. Diante dessa realidade, este trabalho tem como objetivo propor um ambiente para engenharia de requisitos integrado à gerência de processos de negócios, para projetos de pequeno porte. O ambiente proposto, denominado E-flow Project, é composto por um processo para engenharia de requisitos baseado no Rational Unified Process (RUP) e uma ferramenta para automatizar e gerenciar esse processo.
\end{abstract}

\section{Introdução}

O software tem se tornado cada vez mais necessário para as organizações sobreviverem no mercado de uma forma em geral considerado virtualmente inevitável no mundo moderno. Além disso, contempla necessidades dos usuários com requisitos de complexidade cada vez maiores (PRESSMAN, 2002).

A crise do software é conhecida como um reflexo da incapacidade das organizações que atuam com desenvolvimento de software em atender plenamente às necessidades de seus clientes, gerando insatisfação dos seus usuários e em prejuízos financeiros de enormes proporções. Atualmente, cerca de $70 \%$ dos investimentos da área são realizados com o objetivo de manter produtos desenvolvidos anteriormente (REIS, 2002).

Na opinião de Paula Filho (2003), uma das causas para a crise do software está relacionada ao levantamento e a documentação dos requisitos do produto. Quando a documentação é bem feita, os requisitos documentados têm maiores chances de serem corretamente entendidos pelos desenvolvedores. Tal necessidade poderá ser atendida através da engenharia de requisitos, que fornece um mecanismo adequado para entender as expectativas do cliente, analisar as necessidades, avaliar a exeqüibilidade, negociar 
uma solução razoável, especificar a solução de maneira não-ambígua, validar a especificação e administrar os requisitos à medida que eles são transformados num sistema em operação (Somerville apud PRESSMAN, 2002).

Sendo o desenvolvimento de software um trabalho cooperativo entre pessoas, que implica em alta integração de ferramentas e alto nível de descrição das tarefas que compõem as atividades do processo, identifica-se a necessidade de prover um ambiente de desenvolvimento que integre ferramentas, pessoas e o processo (MANZONI, 2001).

O objetivo deste trabalho é propor um ambiente para a engenharia de requisitos integrado a uma solução Business Process Management (BPM). É decomposto em:

- definir um processo para engenharia de requisitos baseado no RUP para pequenos projetos, identificando seus papéis, atividades e artefatos;

- desenvolver uma ferramenta que gerencie e automatize a execução do processo, armazenando e gerenciando as informações capturadas ao longo do processo proposto.

A relevância deste trabalho consiste em oferecer às organizações que atuam com projetos de software de pequeno porte, um ambiente para engenharia de requisitos, orientando quanto aos passos necessários e quais informações deverão ser coletadas e gerenciadas ao longo do processo de desenvolvimento de software. Apesar da existência de várias ferramentas para engenharia de requisitos não foram encontradas soluções que adotassem a mesma abordagem.

\section{Ambiente E-FLOW PROJECT}

Nesta seção é apresentada uma visão sobre os principais objetivos deste trabalho referenciando a metodologia adotada. Posteriormente, é detalhado o processo proposto para engenharia de requisitos contemplando os papéis, atividades e artefatos. Logo após, é exposta a especificação da ferramenta E-flow Project que apoia a execução do processo.

O trabalho iniciou-se com o estudo do RUP focando na disciplina de Requisitos e dos fundamentos da Engenharia de Requisitos para definição do processo de requisitos voltado a projetos de pequeno porte. Foi considerado como projeto de pequeno porte aquele que constituído de 3 a 10 pessoas e que possuem duração inferior a um ano (MANZONI, 2001). O processo definido baseou-se no RUP por se tratar de um modelo amplamente conhecido pela comunidade de software, por ele possuir flexibilidade para customização e por ser fundamentado nas melhores práticas de desenvolvimento de software. Além do RUP, utilizou-se como base para definição do processo os conceitos da Engenharia de Requisitos.

A customização do RUP foi necessária por se tratar de um processo genérico que possui uma grande quantidade de artefatos, papéis e atividades muitas vezes desnecessárias e burocráticas para realidade de algumas organizações (MANZONI, 2001). Para customização do RUP, seguiu-se um roteiro fornecido pelo próprio framework com a preocupação de manter seus princípios baseados nas melhores práticas e buscando identificar as principais atividades e artefatos para capturar os requisitos e gerenciá-los durante o projeto. 
Após a definição do processo, utilizou-se o estudo das ferramentas de Engenharia de Requisitos e trabalhos acadêmicos relacionados com o tema, visando definir os requisitos para a solução E-flow Project que será utilizada para comportar o processo. A etapa posterior consistiu na seleção da ferramenta BPMS. A ferramenta escolhida para automatizar e gerenciar o processo proposto foi o componente opensource e-flow (COSTA, 2004). Para avaliar o ambiente proposto foram utilizadas as diretrizes para customização do RUP e os cinco níveis de maturidade proposto por Heumman (2003).

\subsection{Processo E-FLOW PROJECT}

O processo proposto para Engenharia de Requisitos, chamado processo de requisitos Eflow Project, foi definido a partir da customização do RUP e atividades de alto nível apresentadas na engenharia de requisitos e aplica-se no desenvolvimento de projetos de software de pequeno porte. A sua concepção teve como premissa adaptá-lo para que seja o mais simples possível, mantendo as melhores práticas.

O processo ER, como o processo de requisitos E-flow Project será chamado a partir deste ponto, foi concebido de forma a possibilitar a captura e acompanhamento dos requisitos ao longo do projeto permitindo que os mesmos pudessem ser desenvolvidos utilizando o modelo de processo iterativo e incremental.

Os artefatos gerados pelo processo ER ao longo das atividades são baseados nos templates propostos pelo RUP como é mostrado a seguir:

- documento de visão: neste artefato é registrado principalmente o problema que o software se propõe a resolver, os envolvidos no projeto, e suas necessidades;

- especificação de requisitos: neste documento encontram-se os detalhamentos dos requisitos funcionais orientado a casos de uso e os requisitos não-funcionais;

- glossário: apresenta a lista dos termos utilizados no projeto;

- protótipo de interface do usuário: refere-se ao protótipo de telas do software.

A figura 1 exibe o fluxo de atividades definido para o processo, que mostra a ordem em que as atividades deverão ser executadas. Os responsáveis pela execução de cada uma das atividades são:

- gerente de projetos: este papel é responsável por iniciar o processo ER;

- cliente: este papel é responsável por aprovar os artefatos gerados neste processo;

- analista de sistemas: papel responsável em interagir com o cliente, estabelecendo uma interface entre o cliente e os desenvolvedores do sistema;

- projetista de interface: papel responsável em criar o protótipo de telas do sistema, muitas vezes conhecido como designer. 


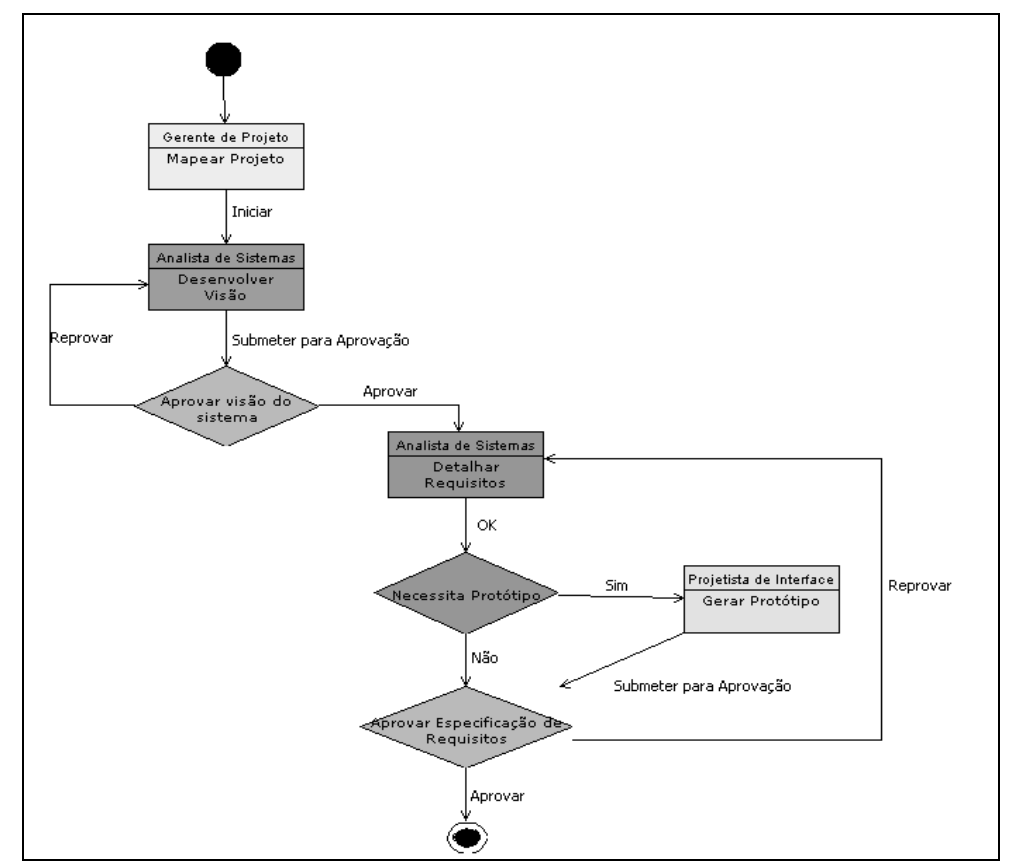

Figura 1. Diagrama de atividades do processo ER.

Para facilitar o entendimento das atividades propostas utilizou-se os elementos visuais do RUP para indicar o papel responsável pela atividade, os artefatos que são visualizados ou alterados durante a atividade e os passos para execução da mesma.

$\mathrm{Na}$ atividade Mapear projeto o gerente de projeto inicia o processo ER informando qual o projeto que será associado à instância do processo. A atividade seguinte, Desenvolver Visão, tem como objetivo desenvolver o artefato de visão e manter o mesmo atualizado. Ela é uma compilação da atividade de elicitação da Engenharia de Requisitos com as seguintes atividades do RUP: "estabelecer acordo sobre o problema”, "identificar os envolvidos" e "identificar solicitações dos principais envolvidos”.

Nessa atividade, o analista de sistemas estabelece uma definição comum sobre o problema que o sistema se propõe a resolver, identifica os envolvidos e obtém suas necessidades priorizando-as. Neste projeto, as necessidades dos envolvidos serão categorizadas em requisitos funcionais e não funcional. Nessa atividade o glossário do sistema é incrementado, consultado e/ou alterado. Após ser estabelecido a visão do sistema, o artefato documento de visão é submetido à aprovação, como mostra a figura 2.

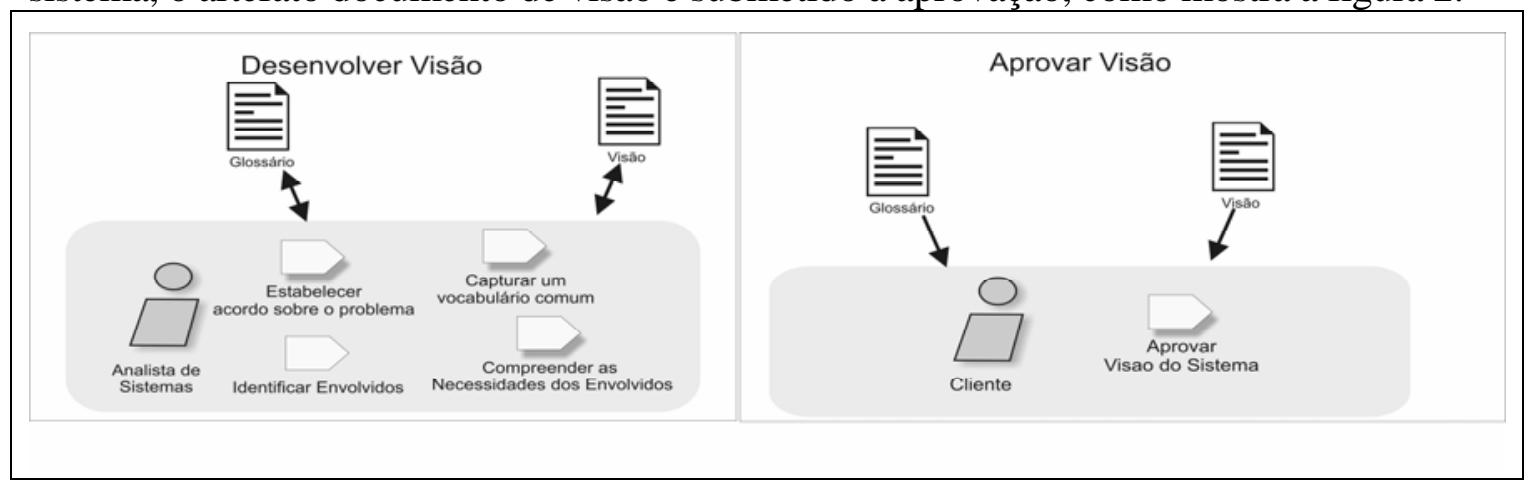

Figura 2. Manter visão. 
Na figura 2 também é possível visualizar a atividade Aprovar visão do sistema. Nesta o cliente é responsável pela aprovação do documento de visão do sistema, verificando os itens de clareza e ambigüidade.

Com a visão aprovada pelo cliente, o analista de sistemas tem a responsabilidade de detalhar os requisitos levantados inicialmente executando a atividade Detalhar requisitos de software. Esta atividade visa refinar os requisitos suplementares identificados na etapa de desenvolver visão e identificar e desenvolver um conjunto de casos de uso para detalhar os requisitos funcionais.

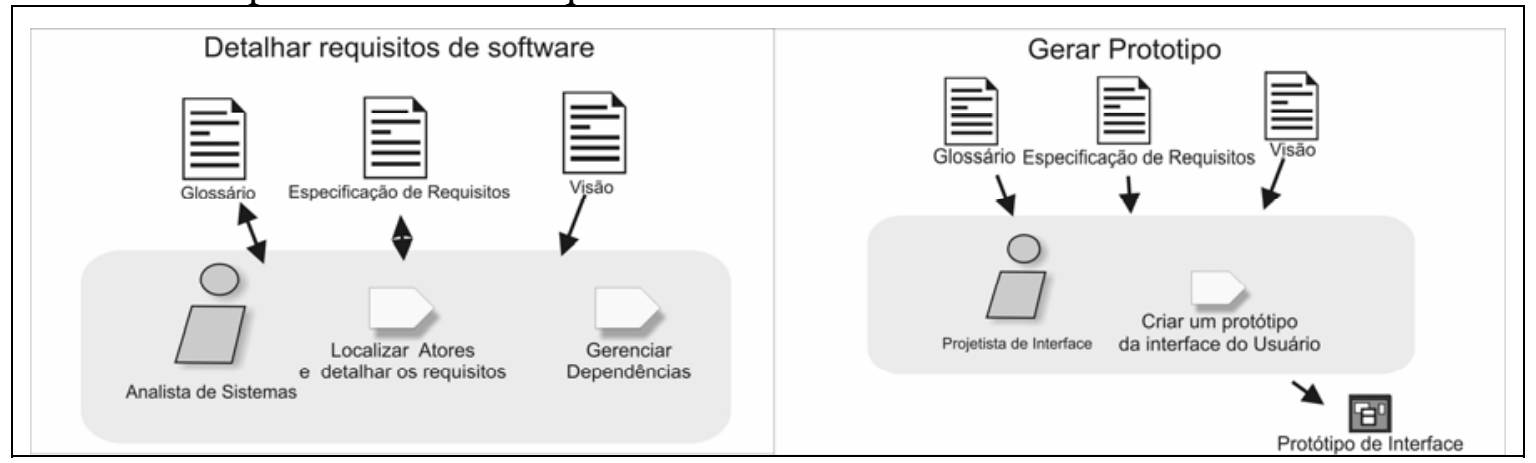

Figura 3. Detalhamentos dos Requisitos.

Como mostra a figura 3, os artefatos manipulados são: i) documento de visão, para consultar os requisitos definidos e já validados pelo cliente; ii) glossário, necessário para gerar o artefato; iii) especificação de requisitos, fruto da atividade. Essa atividade foi desenvolvida tomando por base as atividades "Macro Análise" e "Especificação dos Requisitos" da Engenharia de Requisitos e nas seguintes atividades do RUP: Localizar Atores e casos de uso, Detalhar os requisitos do software, Gerenciar dependência e Definir a especificação suplementar.

Já na atividade Necessita de Protótipo, com os requisitos detalhados, o analista de sistemas verificará a especificação dos requisitos analisando seu grau de clareza. Caso perceba-se a necessidade de protótipo de telas, o processo é direcionado para a atividade Gerar Protótipo.

Na atividade Gerar Protótipo, tendo acesso aos artefatos especificação de requisitos, visão e glossário, como mostra a figura 3, o projetista de interface elabora o protótipo de telas para o sistema ou somente para alguns casos de uso, que se deseja esclarecer. Após gerar o protótipo o processo é encaminhado para atividade de aprovar especificação de requisitos.

$\mathrm{Na}$ atividade Aprovar especificação de requisitos o cliente homologa a especificação dos requisitos para aprovação de todos os elementos funcionais descritos ao longo deste processo. Caso identifique algo não conforme, o cliente deverá reprovar o artefato descrevendo o motivo. Sendo aprovado este processo é finalizado.

As atividades de Aprovar Documento de Visão e Aprovar Especificação de Requisitos são utilizadas como marcos para validação dos artefatos gerados ao longo do processo. 


\subsection{Ferramenta E-FLOW PROJECT}

O objetivo desta seção é apresentar a solução proposta para apoiar a execução e automatização do processo proposto para o ambiente. A solução BPMS escolhida, para atender ao requisito de integração a uma ferramenta BPM foi o software Eflow(COSTA, 2004), pois este atende e apresenta as seguintes características:

- baseado nos padrões da WFMC, porém sendo uma simplificação destes padrões, o que facilitou na compreensão de seu funcionamento;

- utiliza um conjunto de frameworks como o struts e hibernate que padronizam e facilitam sua solução, pois caso a curva de aprendizagem fosse alta inviabilizaria a sua utilização;

- utiliza como motor de processos, o JBPM (Java Business Process Managment), um framework mantido atualmente pelo JBOSS que oferece maior credibilidade ao produto.

Dentro da macro arquitetura do E-flow, os seguintes módulos são destacados:

- JBPM: o E-flow possui o componente Java Business Process Management (JBPM) como base para sua implementação, que por sua vez é baseada na especificação da WfMC (COSTA,2004). O JBPM é utilizado para o gerenciamento e execução dos processos;

- documentação da qualidade: este módulo é uma extensão do JBPM que define uma metalinguagem para apoiar a estruturação dos documentos da qualidade. A extensão consiste na adição de atributos específicos para os documentos da qualidade (COSTA, 2004);

- editor de processo: componente que permite a modelagem do processo, inclusive informando atributos utilizados no módulo de documentação da qualidade e desenvolvimento do fluxo de atividades (COSTA, 2004).

- project: Este módulo foi adicionado ao E-flow, nota-se neste módulo a existência de vários módulos, dentre esses destaca-se o módulo reqSystem, responsável em armazenar e disponibilizar as informações adquiridas ao longo do processo proposto para Engenharia de Requisitos. Os outros módulos foram propostos para automatizar as disciplinas apresentadas no RUP (a implementação destes módulos é uma sugestão para trabalhos futuros).

\section{Avaliação dos Resultados}

Para avaliação dos resultados obtidos neste trabalho, o ambiente E-flow Project foi submetido a duas avaliações. A primeira avaliação consistiu em verificar quais diretrizes propostas pelo RUP, para customização do framework, foram contemplados no ambiente proposto. Na segunda avaliação, o e-flow project foi classificado dentro dos cinco níveis de maturidade de gerenciamento de requisitos proposto por Heumann (2003).

A tabela 1 exibe os resultados apresentados pela primeira avaliação do E-flow Project. Dentre as avaliadas, a diretriz "Usar ferramentas integradas" não foi contemplada e "Manter as melhores práticas" foi contemplada parcialmente devido ao escopo definido para este trabalho. 
Tabela 1 Avaliação dentro das diretrizes do RUP.

\begin{tabular}{|c|c|c|}
\hline DIRETRIZES RUP & $\begin{array}{c}\text { AMBIENTE } \\
\text { E-FLOW PROJECT }\end{array}$ & COMENTARIOS \\
\hline $\begin{array}{l}\text { 1. Manter as melhores } \\
\text { práticas }\end{array}$ & $\begin{array}{l}\text { Contemplado } \\
\text { parcialmente }\end{array}$ & $\begin{array}{l}\text { Visto que o ambiente proposto possui escopo limitado à } \\
\text { engenharia de requisitos, ele contempla apenas a boa } \\
\text { pratica de gerência de requisitos. }\end{array}$ \\
\hline $\begin{array}{l}\text { 2. Não incluir atividades e } \\
\text { artefatos que não possam ser } \\
\text { claramente justificados }\end{array}$ & Foi contemplado & $\begin{array}{l}\text { Todas as atividades e artefatos abordados no processo } \\
\text { foram identificados e justificados. }\end{array}$ \\
\hline $\begin{array}{l}\text { 3. Poupar os desenvolvedores } \\
\text { em relação ao processo }\end{array}$ & Foi contemplado & \\
\hline $\begin{array}{l}\text { Minimizar os artefatos } \\
\text { formais }\end{array}$ & Foi contemplado & \\
\hline $\begin{array}{l}\text { 4. Gerar artefatos } \\
\text { automaticamente }\end{array}$ & Foi contemplado & $\begin{array}{l}\text { Todos os artefatos são gerados automaticamente pela } \\
\text { ferramenta e-flow project a partir de informações } \\
\text { coletadas ao longo do processo }\end{array}$ \\
\hline 5. Usar a Web & Foi contemplado & \\
\hline $\begin{array}{l}\text { 6. Usar ferramentas } \\
\text { integradas }\end{array}$ & Não contemplado & $\begin{array}{l}\text { O E-flow Project contempla apenas a gerência de } \\
\text { requisitos e não está integrado a outras ferramentas de } \\
\text { apoio ao processo de desenvolvimento de software, essa } \\
\text { característica será proposta em trabalhos futuros. }\end{array}$ \\
\hline $\begin{array}{l}\text { 7. Rever o Processo } \\
\text { Regularmente }\end{array}$ & Foi contemplado & $\begin{array}{l}\text { O ambiente não determina a periodicidade de revisão do } \\
\text { processo, entretanto permite deixando essa } \\
\text { responsabilidade para o analista de processos. }\end{array}$ \\
\hline
\end{tabular}

\section{Considerações finais}

Este trabalho propôs um ambiente para Engenharia de Requisitos integrado a Gerência de Processos de Negócios (BPM), denominado E-flow Project. Para conceber o ambiente, foi definido um processo para Engenharia de Requisitos baseado no RUP e desenvolvida uma ferramenta para gerenciar e automatizar o processo.

As avaliações às quais foi submetido o ambiente E-flow Project evidenciam resultados satisfatórios na proposta do ambiente para a Engenharia de Requisitos. Além das vantagens identificadas, deve ser considerado que a não utilização do E-flow Project em ambiente colaborativo, poderá tornar o processo custoso provocando desuso do ambiente.

No decorrer do desenvolvimento deste trabalho foram encontradas as seguintes dificuldades:

- proposta de processo: condensar todo o conjunto de conceitos (Engenharia de Requisito e RUP) e metodologia em um ambiente para apoiar a gerência de requisitos em pequenos projetos;

- definição da arquitetura da solução: entender toda a especificação, projeto e implementação da ferramenta BPMS para que ela pudesse ser usada para comportar o reqSystem e para fazer alguns ajustes para que o objetivo deste trabalho fosse contemplado.

Para continuidade deste trabalho, foram identificados alguns pontos que poderão ser melhorados ou ampliados em trabalhos futuros: 
- módulos do E-flow Project: implementar os outros módulos no E-flow Project para se ter uma ferramenta que contemple todas as disciplinas do RUP;

- interface de integração: definir interfaces de integração entre ferramentas de apoio ao desenvolvimento de software com o E-flow Project usando SOA;

- experimento prático: implantar o E-flow Project em uma organização para avaliar o ambiente em um projeto de software de pequeno porte;

\section{Referências}

COSTA, Victor Franco. E-flow: uma solução de workflow para integração da gestão de processos com a documentação da qualidade usando xml. 2004. 75 f. Monografia (Bacharel em Informática) - Curso de bacharelado em Informática, Universidade Católica do Salvador.

HEUMANN, Jim. The Five Levels of Requirements Management Maturity. Rational Edge. 2003. Disponível em: <http://www128.ibm.com/developerworks/

rational/library/content/RationalEdge/feb03/ManagementMaturity_TheRationalEdge_F eb2003.pdf>. Acesso em: 22 dez. 2005.

MANZONI, Lisandra Vielmo. Uso de Sistema de Gerência de Workflow para Apoiar o Desenvolvimento de Software Baseado no Processo Unificado Rational Estendido para Alcançar Níveis 2 e 3 do Modelo de Maturidade. 2001. Dissertação (Mestrado) - Programa de Pós-Graduação em Computação, Universidade Federal do Rio Grande do Sul, Porto Alegre, 2001.

PAULA FILHO, Wilson de Pádua. Engenharia de Software: Fundamentos, Métodos e Padrões. 2. ed. Rio de Janeiro: LTC, 2003.

PRESSMAN, Roger S. Engenharia de Software. 5. ed. Rio de Janeiro: McGraw-Hill, 2002. 843 p.

REIS, Rodrigo Quites; et al. Automação no Gerenciamento do Processo de Engenharia de Software. [artigo científico]. Disponível em: < http://www.ulbrato.br/ensino/43020/artigos/anais2002/EIN2002/ProcessoEngenhariaSoftware.pdf>.

Acesso em: 27 abr. 2006. 